\title{
Experimental studies on the photoelectron instability in the Beijing Electron Positron Collider
}

\author{
Z. Y. Guo, H. Huang, S. P. Li, D. K. Liu, L. Ma, Q. Qin, J. Q. Wang, S. H. Wang, G. Xu, C. Zhang, and Z. Zhao \\ Institute of High Energy Physics, Chinese Academy of Sciences, Beijing 100039, People's Republic of China \\ Y. H. Chin, H. Fukuma, S. Hiramatsu, M. Izawa, T. Kasuga, E. Kikutani, Y. Kobayashi, S. Kurokawa, K. Ohmi, Y. Sato, \\ Y. Suetsugu, M. Tobiyama, K. Tsukamoto, K. Yokoya, and X. L. Zhang \\ High Energy Accelerator Research Organization, Tsukuba-shi, Ibaraki-ken, 305-0801, Japan
}

(Received 29 March 2002; published 27 December 2002)

\begin{abstract}
A vertical coupled-bunch instability was observed for a positron beam at the Beijing Electron Positron Collider (BEPC). The experimental results show that the instability has similar characteristics as that observed in the Photon Factory of KEK several years ago. The instability at BEPC can be explained by the effect of an electron cloud which is produced in the beam chamber by synchrotron light hitting the wall.
\end{abstract}

DOI: 10.1103/PhysRevSTAB.5.124403

PACS numbers: 29.20.Dh, 29.27.Bd

\section{INTRODUCTION}

Several years ago a vertical coupled-bunch instability with a low threshold current was observed in the Photon Factory (PF) at KEK when the machine was operated with a multibunch positron beam [1]. According to the physical model proposed, the instability was caused by a cloud having an enormous number of electrons produced by synchrotron light hitting the inner surface of the beam chamber [2]. The number of photons due to the synchrotron radiation emitted by a positron per meter is expressed by

$$
N_{\gamma}\left[/\left(m \cdot e^{+}\right)\right]=\frac{5 \pi}{\sqrt{3}} \frac{\alpha \gamma}{L},
$$

where $\alpha, \gamma$, and $L$ are the fine structure constant, the relativistic factor, and the circumference of the ring, respectively. Photoelectrons are produced by photons at the chamber surface with a quantum efficiency around $\sim 0.1$. The number of photoelectrons produced by a positron per meter is $0.01 \sim 0.1 /\left(m \cdot e^{+}\right)$in most of the positron rings, although this depends on the ring parameters. The photoelectrons are accumulated in the beam chamber up to several or 10 times the number produced for a bunch population $\left(\sim 10^{10}\right)$ with a narrow bunch repetition $(\sim 1 \mathrm{~m})$. Therefore the neutralization factor, which is the ratio of the line density of the photoelectrons to that of the positron beam, can be close to 1 . The electron cloud induces a wake force as a result of the passage of the bunch train, which causes a coupled-bunch instability. Because of this mechanism, we refer to it as photoelectron instability (PEI).

This instability is a serious problem for all of the high intensity positron rings, namely, for our present and future accelerator projects, B and $\tau$-charm factories, and positron damping rings in linear colliders.

In order to understand the nature of PEI, a series of experiments have been carried out at the Beijing Electron
Positron Collider (BEPC) since 1996 under the cooperation of IHEP in China and KEK in Japan [3]. In the experiments a vertical coupled-bunch instability was observed for a positron beam at approximately $10 \mathrm{~mA}$ of beam current. A broad distribution of the vertical betatron sidebands appeared when the instability occurred. We then studied the dependence of the instability on several beam parameters, such as the chromaticity, bunch spacing, emittance, beam energy, $r f$ frequency, on/off status of distributed ion pumps, betatron tunes, magnetic field, and rf voltage. During an experiment in 1998, we used a single-pass beam position monitor system (SPBPM) to analyze the oscillation in the time domain and investigated the effect of an octupole field on the instability. To examine whether the observed instability can be explained by the PEI model, a computer code was developed to simulate the instability process. The experimental results were compared with the simulations. We report on these experimental and simulation studies in this paper.

\section{EXPERIMENTAL RESULTS}

BEPC has been operated as an electron positron collider as well as a synchrotron radiation source with a maximum single beam current of $150 \mathrm{~mA}$ at beam energies between $E=1.3$ and $2.2 \mathrm{GeV}$. The main parameters of the BEPC under the typical experimental operation are $E=1.3 \mathrm{GeV}$, betatron tune $\nu_{x}=5.82$ in the horizontal direction, $\nu_{y}=6.74$ in the vertical direction, natural emittance $\varepsilon_{x}=0.134 \mu \mathrm{m} \mathrm{rad}$, rf frequency $f_{\mathrm{rf}}=200 \mathrm{MHz}$, transverse damping time of $86 \mathrm{~ms}$, harmonic number $h=160$, and minimum bunch spacing of $5 \mathrm{~ns}$. It can be operated with various beam emittance by changing the optics and can also be operated in various filling patterns of bunches. The beam chamber of the BEPC is made of aluminum and has a cross section of $58 \mathrm{~mm}$ in height and $120 \mathrm{~mm}$ in width. 
A spectrum analyzer with a $1.5 \mathrm{GHz}$ bandwidth connected to a button pickup was used to observe the mode spectrum of the instability. A synchrotron light monitor was used to measure the transverse beam profile. A wallcurrent monitor was used to check the relative population of particles in each rf bucket and to detect the bunch filling pattern. The SPBPM system was provided by KEK to observe the beam oscillation [4]. It enabled us to acquire data in a very short time and to study the time development of the instability.

The first experiment on the PEI was performed in June, 1996. The conditions of the experiment were $E=$ $1.55 \mathrm{GeV}, \nu_{x}=5.79, \nu_{y}=6.71, \varepsilon_{x}=0.194 \mu \mathrm{m} \mathrm{rad}$, and chromaticities $\xi_{x}=4.0, \quad \xi_{y}=4.0$. Positrons were injected almost uniformly into 158 buckets with a total beam current of $16.6 \mathrm{~mA}$. Vertical betatron sidebands, $m f_{0} \pm f_{\beta}$, were observed on the spectrum analyzer, as shown in Fig. 1, where $f_{\beta}$ is the vertical betatron frequency and $f_{0}$ the revolution frequency. The positive and negative values on the $y$ axis correspond to $m f_{0}+f_{\beta}$ and $m f_{0}-f_{\beta}$, respectively. The vertical oscillation was also observed with the synchrotron light monitor.

The figure shows that the instability is caused by a coupled-bunch oscillation. The distribution of the vertical betatron sidebands is broad. We did not find any betatron sidebands caused by higher order modes at the corresponding frequency in the rf cavities. The growth time of the transverse resistive wall instability $(\sim 1.55 \mathrm{~s}$ at $1.55 \mathrm{GeV}$ ) in the BEPC is much longer than the synchrotron radiation damping time $(50 \mathrm{~ms}$ at $1.55 \mathrm{GeV})$.

A series of experiments was then carried out after the first observation of the instability. The instability was easily reproduced under similar beam conditions. The

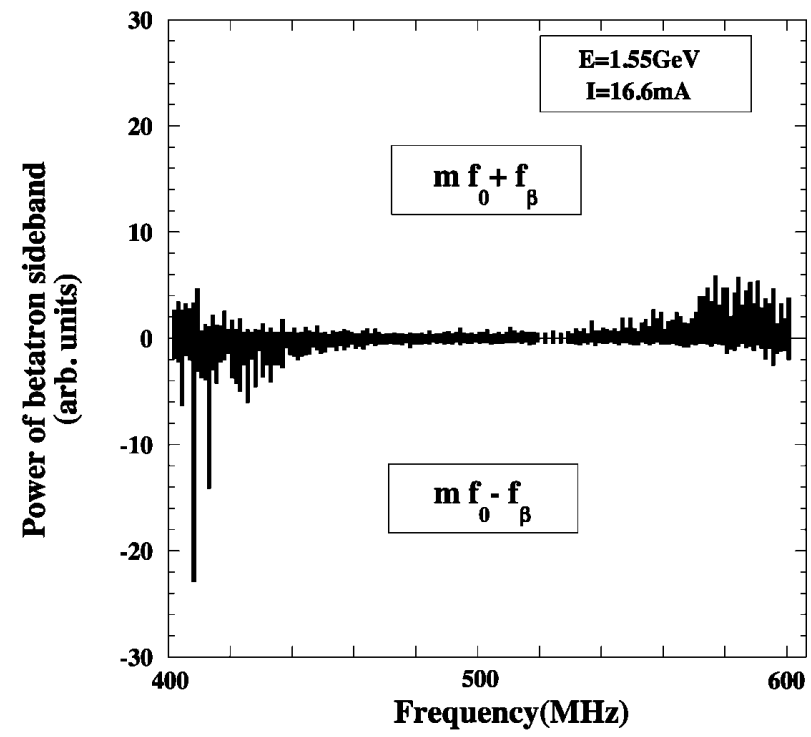

FIG. 1. Vertical betatron sidebands at a beam energy of $1.55 \mathrm{GeV}$. The positive and negative values correspond to $m f_{0}+f_{\beta}$ and $m f_{0}-f_{\beta}$, respectively. threshold current of the instability was around $9.3 \mathrm{~mA}$ at $1.3 \mathrm{GeV}$ with 160 uniformly filled bunches.

The effects of the machine parameters on the PEI were studied. The instability strongly depended on the bunch spacing. The threshold current of the instability was higher than $40 \mathrm{~mA}$ when the positrons were injected into every two buckets.

The threshold also depended on the chromaticity. At a beam current of $9.4 \mathrm{~mA}$ with 160 bunches the vertical betatron sidebands disappeared when the vertical chromaticity $\left(\xi_{y}\right)$ changed from 4 to 6 and reappeared when the chromaticity returned to 4 . At a current of $38.2 \mathrm{~mA}$ with 80 uniformly filled bunches the vertical betatron sidebands were observed when the vertical chromaticity $\left(\xi_{y}\right)$ changed from 4 to 2.8 and disappeared when the chromaticity returned to 4 .

The effect of the emittance on the instability was investigated at two optics with emittances of 0.024 and $0.14 \mu \mathrm{mrad}$. In the optics with an emittance of $0.024 \mu \mathrm{mrad}$ the tunes and chromaticities were $\nu_{x}=$ 9.38, $\nu_{y}=5.14, \xi_{x}=1.0$, and $\xi_{y}=1.5$. The threshold current of the instability with 160 uniformly filled bunches was about $9 \mathrm{~mA}$ at an emittance of $0.14 \mu \mathrm{m} \mathrm{rad}$ and $16 \mathrm{~mA}$ at $0.024 \mu \mathrm{m} \mathrm{rad}$, respectively.

To survey the energy dependence of the instability, the beam energy was scanned from 1.3 to $2.2 \mathrm{GeV}$ at a beam current of about $10 \mathrm{~mA}$ with 160 uniformly filled bunches. The amplitude of the vertical betatron sidebands did not change at energies of between 1.3 and $1.85 \mathrm{GeV}$. It slightly decreased at $2.0 \mathrm{GeV}$ even though there were still a few modes with considerable strength. At $2.2 \mathrm{GeV}$ the vertical betatron sidebands disappeared. Figure 2 shows the sideband spectrum at $2.0 \mathrm{GeV}$.

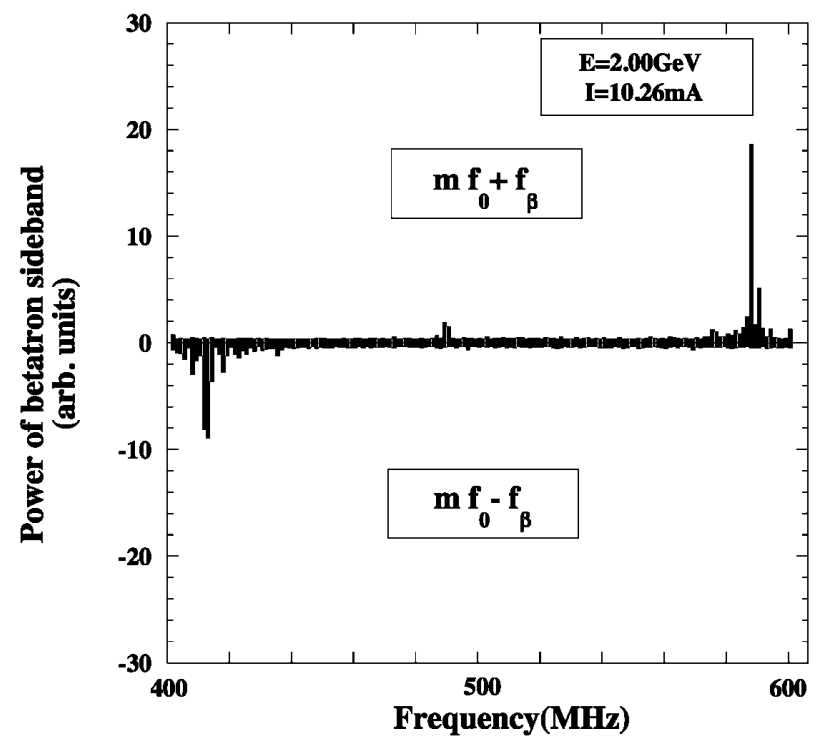

FIG. 2. Vertical betatron sidebands at a beam energy of $2.0 \mathrm{GeV}$. The positive and negative values correspond to $m f_{0}+$ $f_{\beta}$ and $m f_{0}-f_{\beta}$, respectively. 


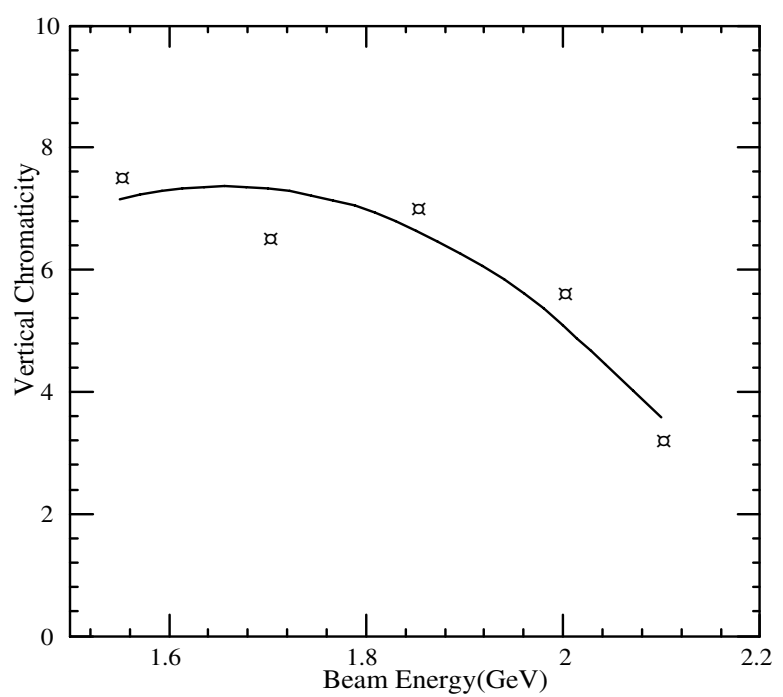

FIG. 3. Energy dependence on the chromaticity at the threshold value.

The vertical chromaticity was changed at different energies to obtain the threshold value at which the sidebands disappear. The relation between the beam energy and the vertical chromaticity at the threshold value is shown in Fig. 3.

The observation showed that the transverse tunes did not affect the vertical betatron sidebands of the instability when they were surveyed in a stable region $\left(\Delta \nu_{x}, \nu_{y} \sim\right.$ \pm 0.05 ). To see the effect of the synchrotron tune on the instability, the $\mathrm{rf}$ voltage was changed from 0.25 to $0.8 \mathrm{MV}$ (0.3 MV in normal operation). There was no influence of the rf voltage on the vertical betatron sidebands of the instability.

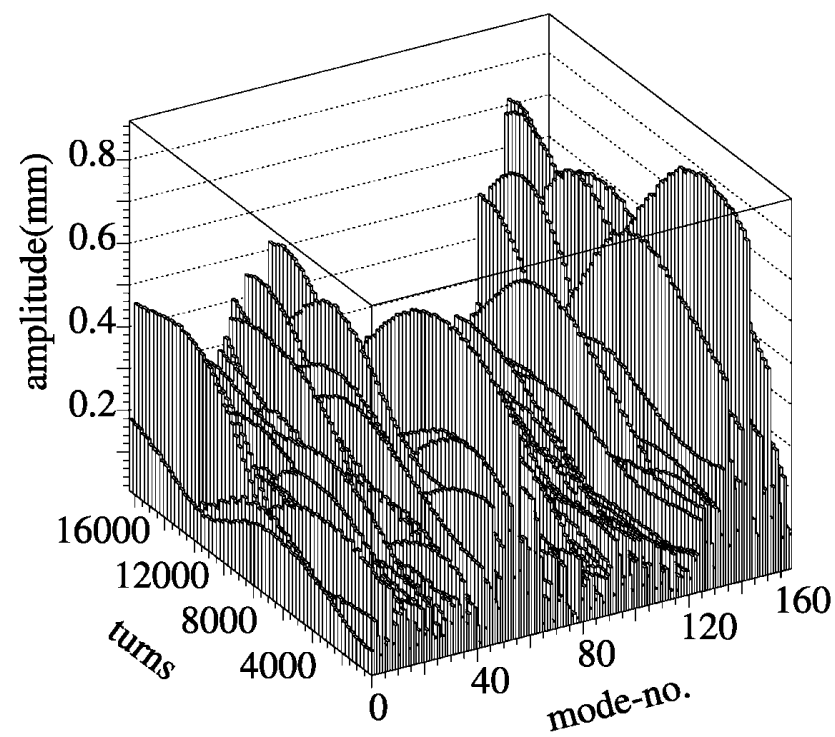

It was reported in the Cornell Electron Storage Ring that the leakage electrostatic field from the distributed ion pump traps photoelectrons, and then the photoelectron instability could have resulted. There are 40 distributed ion pumps in the bending magnets along the BEPC ring. The distribution of the vertical betatron sidebands was compared in the case of the pumps being turned on and off. The characteristics of the instability were not changed significantly.

A completely different distribution of the betatron sidebands was observed while operating the electron beam under the same conditions as the positron beam. In the filling of $9.8 \mathrm{~mA}$ with 160 bunches, the vertical sidebands were observed only near the rf frequency $\left(f_{\mathrm{rf}}\right)$ and almost no vertical betatron sidebands were observed at other revolution harmonics. In the filling of $38 \mathrm{~mA}$ with 80 bunches uniformly distributed, both the vertical and horizontal betatron sidebands were observed only near $f_{\text {rf }}$, and no betatron sidebands were observed at the other revolution harmonics.

In 1998, SPBPM was introduced in the experiment. SPBPM enabled us to acquire the transverse position of all bunches during 16384 turns to study the time development of the instability. We investigated the mode spectra as a function of time by a mode analysis. The whole 16384-turn data was divided into 64 sets of successive 256-turn subdata, and mode analysis was performed for each subdata. Combining these 64 mode spectra, we obtained the behavior of the modes over 16384 turns, as shown in Fig. 4. The results confirmed the mode spectra obtained by the spectrum analyzer and showed a prominent difference of the instabilities in the positron and electron beams.

An octupole magnet was installed in BEPC to observe its effect on the instability. When the octupole was

FIG. 4. Behavior of the modes for positron (left panel) and electron (right panel) bunches. 
excited to $K_{3}=e B^{\prime \prime \prime} L / p=33 \mathrm{~m}^{-3}$, the instability was suppressed. The Landau damping time by exciting the octupole magnet is estimated to be $6.2 \mathrm{~ms}$.

\section{SIMULATIONS AND COMPARISON WITH THE EXPERIMENTS}

The characteristics of the observed instability were compared with both theoretical and simulation studies based on the PEI model. The instability was analyzed using a wake force, which is induced by the photoelectron cloud [2]. The photoelectrons are produced at the inner surface of the beam chamber and propagate under the influence of the electric force of the positron beam. An electron cloud is formed in the beam chamber due to photoelectron productions at the successive bunch passages with the repetition. The wake force induced by the electron cloud is obtained by a numerical method: (i) we first calculate a stationary photoelectron distribution (cloud), (ii) we then apply a perturbation to the cloud passing a bunch with a transverse displacement $\left(\Delta y_{0}\right)$, and (iii) finally we calculate the total velocity kicks $\left(\Delta v_{y, k}\right)$ that electrons experience due to successive bunches denoted by $k$. The velocity kick divided by the displacement is related to the wake field as follows:

$$
N_{p}^{2} r_{e} c W(z=k L / h)=\frac{d v_{y, k}}{d y_{0}},
$$

where $N_{p}$ is the number of positrons in a bunch. The growth rate is calculated by the dispersion relation with the assumption of a linear wake expressed as [6]

$$
\Omega_{m}-\omega_{\beta}=\frac{1}{4 \pi \gamma \nu_{y} N_{p}} \sum_{k=1}^{n} \frac{d v_{y, k}}{d y_{0}} e^{2 \pi i k\left(m+\nu_{y}\right) / h},
$$

where $N_{e}$ is the number of photoelectrons produced by a bunch through the ring circumference, $N_{p}$ the number of positrons in a bunch, $n$ the range of the wake force, and $\gamma$ the Lorentz factor. $d v_{y, k} / d y_{0}$ is the wake force for the $k$ th bunch due to the displacement of a bunch $\left(y_{0}\right)$ in units of the velocity kick of the photoelectron cloud divided by the displacement.
The electron cloud distribution and the wake force have been studied for the BEPC [3]. The vertical sidebands obtained by the wake force model are shown in Fig. 5(a). The spectrum was similar to those of the experiments. The growth time was estimated to be $10 \mathrm{~ms}$. The relation between the growth time and the bunch spacing is shown in Fig. 5(b). It is clear that the growth time increases for a larger bunch spacing at $100 \mathrm{~mA}$, while it is not clear at $10 \mathrm{~mA}$. The beam-photoelectron interaction has the nature of a short-range wake force. The experimental results, in which the instability becomes weak for a bunch spacing of $10 \mathrm{~ns}$, can be understood qualitatively, but did not quantitatively coincide with the simulation.

A rigid bunch simulation method for the beam-photoelectron interaction was applied to track the coherent oscillation of the bunches [7]. In this method, the photoelectrons are described by macroparticles, and the positrons are described by a series of rigid Gaussian bunches. The photoelectron distribution is assumed to be uniform in the longitudinal direction. The equations of the transverse motion are expressed as follows:

$$
\frac{d^{2} \boldsymbol{x}_{p}}{d s^{2}}+K(s) \boldsymbol{x}_{p}=-\frac{2 r_{e}}{\gamma} \sum_{j=1}^{N_{e}} \boldsymbol{F}\left[\boldsymbol{x}_{p}-\boldsymbol{x}_{e, j} ; \sigma(s)\right] \delta\left(s-s_{j}\right),
$$

$$
\begin{aligned}
\frac{d^{2} \boldsymbol{x}_{e}}{d t^{2}}= & -2 N_{p} r_{e} c \boldsymbol{F}\left[\boldsymbol{x}_{p}-\boldsymbol{x}_{e} ; \sigma(s)\right] \delta\left[t-t\left(s_{j}\right)\right] \\
& -\frac{e}{m_{e}} \frac{\partial \phi\left(\boldsymbol{x}_{e, j}\right)}{\partial \boldsymbol{x}_{e, j}},
\end{aligned}
$$

where indices $p$ and $e$ of $\boldsymbol{x}$ denote the positron and photoelectron, $r_{e}$ the classical electron radius, $m_{e}$ the electron mass, $c$ the speed of light, $e$ the electron charge, $\sigma$ the transverse beam size, $\phi$ the photoelectron potential, and $\boldsymbol{F}$ the Coulomb force in two-dimensional space expressed by the Bassetti-Erskine formula [8].

We calculated the beam motion for $I=100 \mathrm{~mA}$ instead of $I=10 \mathrm{~mA}$ which was the beam current during the experiment, because the growth for $I=10 \mathrm{~mA}$ is too

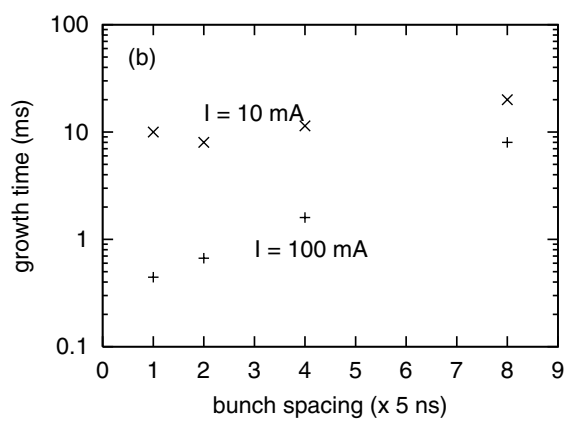

FIG. 5. Results of the wake force method for the BEPC. (a) is the mode spectrum at $I=$ $10 \mathrm{~mA}$, where the negative values mean damping. (b) is the growth time for various bunch spacings at $I=10$ and $100 \mathrm{~mA}$. 

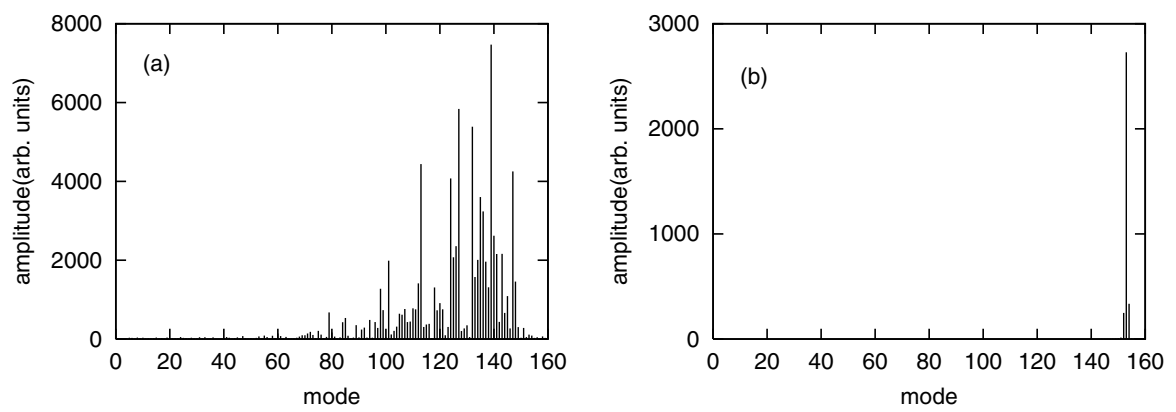

FIG. 6. Sideband of the oscillation simulated at a beam energy of $1.3 \mathrm{GeV}$. (a) and (b) are for positron and electron beams, respectively.

slow in the calculation. The results of tracking show that coherent coupled-bunch oscillation appears along the bunches. The coupled-bunch oscillation obtained from the tracking method was transformed to the spectrum $m$ using a fast Fourier transform. The simulated spectrum is shown in Fig. 6(a) [7]. Similar tracking for an electron beam $(I=10 \mathrm{~mA})$ interacting with ions $\left(\mathrm{CO}^{+}\right)$ gave the result shown in Fig. 6(b). They are consistent with the observed spectra in the experiments. We investigated the growth rate for various emittances and energies. The results showed that there was no significant difference. Note that the growth time remains constant for increasing energy because $d v \propto N_{e} \propto \gamma$ in Eq. (3), if the space charge force between electrons is not dominant.

Next, we discuss the dependence of the chromaticity on the instability. There seems to be little possibility that the instability is affected by the chromaticity, because the beam-electron force does not depend on the chromaticity. We conjecture that the dependence of the chromaticity is due to the damping mechanism. The Landau damping is considered to be the strongest among various damping mechanisms in our experimental conditions for the following reasons: (i) The head-tail damping time was $53 \mathrm{~ms}$ in the calculation under the experimental conditions, $I=$ $62.5 \mu \mathrm{A} /$ bunch and $\xi_{y}=4$; (ii) the radiation damping time was 85 and $23 \mathrm{~ms}$ at $E=1.3$ and $2.0 \mathrm{GeV}$, respectively; (iii) the calculated Landau damping time was about $9.4 \mathrm{~ms}$ under the experiment conditions $\left(\xi_{y}=4\right)$ and 9.9 and $4.9 \mathrm{~ms}$ for $\xi_{y}=6$ and $\xi_{y}=8$ respectively; and (iv) the multibunch effect of the chromatic phase modulation was not strong in the BEPC [9]. The Landau damping time generally becomes faster for a larger chromaticity because sextupole magnets are excited more strongly. In our case, the damping time for $\xi_{y}=4$ is comparable with that for $\xi_{y}=6$, because it depends on the lattice parameters. We note that the Landau damping time is of the same order as that of the calculated growth time of the PEI. Thus, the chromaticity effect may be due to the Landau damping. Otherwise, there may be another mechanism for the chromaticity effect. In either case, since it is well known that the chromaticity works to suppress coupled-bunch instabilities, this experimental result is not strange. We note that the instability was suppressed by the octupole magnet having a similar Landau damping time $(6.2 \mathrm{~ms})$.

We next discuss the dependence of the emittance on the instability. The emittance can affect the instability, because the beam-electron force is a function of it, while our simulations showed that the emittance did not affect the instability. These two facts do not contradict each other. Since the electron cloud distributes throughout the chamber, the integrated force does not depend on the emittance strongly, with the result that the emittance does not affect the instability. Let us try to understand the behavior from the viewpoint of the damping time here. In a low emittance lattice of $\varepsilon_{x}=0.024 \mu \mathrm{m} \mathrm{rad}$, the Landau damping time is estimated to be $7.3 \mathrm{~ms}$ for $\left(\xi_{x}, \xi_{y}\right)=$ $(1,1.5)$, which may explain why the threshold current of the instability in the optics with $\varepsilon_{x}=0.024 \mu \mathrm{m} \mathrm{rad}$ was higher than that in the optics with $\varepsilon_{x}=0.14 \mu \mathrm{m} \mathrm{rad}$.

The instability decreased at a higher energy in the experiments, whereas a clear dependence on energy was not seen in the simulations. The $\gamma$ factor disappears in the formula of the growth rate, because of canceling that in the denominator by the number of photoelectron in the numerator for this case. Let us again discuss the energy dependence from the viewpoint of the damping time. At a higher energy, the emittance increases in proportion to $\gamma^{2}$, which means that the Landau damping time becomes in proportion to $1 / \gamma^{2}$. The growth time of the PEI does not become longer as the energy increases [Eq. (3)], while that of conventional instability is proportional to $\gamma$; that is, the energy dependence of the PEI is weaker than that of the conventional instability. The observed dependence of the instability on the energy can be explained qualitatively based on the above discussion.

This instability model has an energy dependence for the growth rate, when the density of photoelectrons is near the neutralization level. The number of electrons is determined by the neutralization, but is not determined by the production efficiency $(\propto \gamma)$. In this case, the growth rate becomes slower at higher energy. It is difficult to conclude which mechanism is important in our experiments. 


\section{CONCLUSION}

A series of experiments have been carried out to study the PEI at the BEPC. A vertical coupled-bunch instability was observed for a positron beam. The broad distribution of the vertical betatron sidebands was detected in the beam signal by the spectrum analyzer and by the single-pass beam position monitor. For an electron beam, an instability with a narrow betatron sideband distribution was observed. The observed betatron sideband spectra for the positron beam is well explained by a simulation based on the PEI model, while these for the electron beam are well explained by the ion trapping. The characteristics of the positron instability are similar to those of the instability observed in KEK-PF. This instability strongly depended on the bunch spacing, i.e., it was suppressed for longer bunch spacing. This can be explained by taking into account the short-range nature of the beam-photoelectron interaction. This positron instability is understood to be caused by the photoelectron cloud.

We studied the dependence of various beam parameters - the chromaticity, the emittance, and the energyon the positron instability and compared it with the simulation of the PEI model. The instability was suppressed by a higher chromaticity. There is perhaps no special mechanism of photoelectron instability, since it has been generally discussed that the chromaticity suppressed transverse coupled-bunch instabilities. In our case, Landau damping was considered to be a main reason. Our experiments showed that the emittance and energy affect the instability. These parameters may affect qualitative effects in our model, but the effects are not seen in simulations. These dependences on the emittance and energy can be also explained by Landau damping. It is difficult to judge which is dominant, the nature of our PEI mode or Landau damping. The behavior for on/off status of distributed ion pumps and rf voltage, in which the instability was not affected, is reasonable for our model.

We understand that the photoelectron instability occurs in all high intensity positron rings. This result is meaningful to the design and operation of the modern electron positron collider. Antechamber, which is useful to sup- press the production of the photoelectrons, is a common choice for the design of the rings like $\mathrm{B}$ factories and $\tau$-charm factories, as well as the positron damping rings of linear colliders. The surface material and treatments of the beam chamber to reduce the photoelectron and secondary electron emission may be important issues. Attention must be paid to the photoelectron instability at the design stage of the rings. We also need to study this instability from the viewpoint of the beam dynamics.

\section{ACKNOWLEDGMENTS}

The authors are grateful to the BEPC operation team for its cooperation during the experiments. This work was supported by the National Natural Science Foundation of China (19975056) and the Japan Society for the Promotion of Science.

[1] M. Izawa et al., Phys. Rev. Lett. 74, 5044 (1995).

[2] K. Ohmi, Phys. Rev. Lett. 75, 1526 (1995).

[3] Z.Y. Guo et al., in Proceedings of the 1997 Particle Accelerator Conference, Vancouver, edited by M. Comyn et al. (IEEE, Piscataway, NJ, 1997), p. 1566; Z. Y. Guo et al., in Proceedings of the 1998 Asian Particle Accelerator Conference, Tsukuba, edited by Y. H. Chin et al. (KEK Report No. 98-10, 1998), p. 432; Z. Y. Guo et al., in Proceedings of the 1999 Particle Accelerator Conference, New York, edited by A. Luccio and W. MacKay (IEEE, Piscataway, NJ, 1999), p. 633.

[4] M. Tobiyama and E. Kikutani, Phys. Rev. ST Accel. Beams 3, 012801 (2000).

[5] T. Holmquist and J. T. Rogers, Phys. Rev. Lett. 79, 3186 (1997).

[6] A. Chao, Physics of Collective Beam Instabilities in High Energy Accelerators (Wiley-Interscience, New York, 1993.

[7] K. Ohmi, Phys. Rev. E 55, 7550 (1997); in Proceedings of the 1997 Particle Accelerator Conference, Vancouver (Ref. [3]), p. 1667.

[8] M. Bassetti and G. Erskine, CERN Report No. CERN/ ISR/TH/80-06, 1980.

[9] K. Ohmi, KEK Report No. 97-9, 1997, IHEP/BEPC/AP/ Report No. 97-09, 1997. 